NISTIR 7487

\title{
Manufacturing Metrology and Standards for the Health Care Enterprise Program Summary
}

Ram D. Sriram and Steven J. Fenves, Editors Manufacturing Systems Integration Division Manufacturing Engineering Laboratory

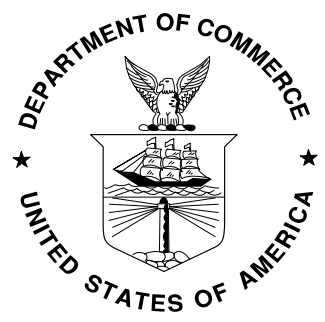

U.S. Department of Commerce Carlos M. Gutierrez, Secretary 


\section{TABLE OF CONTENTS}

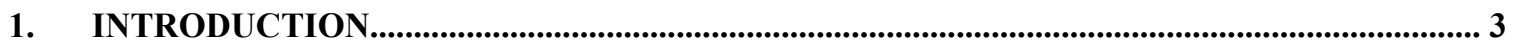

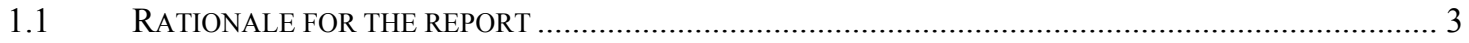

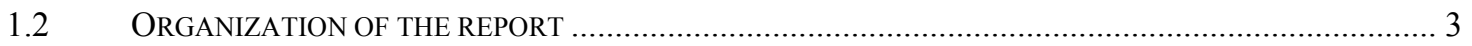

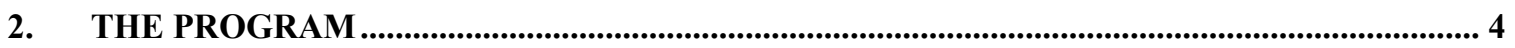

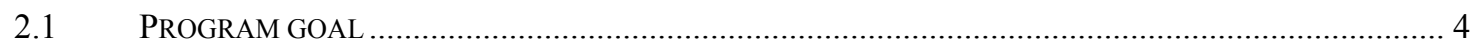

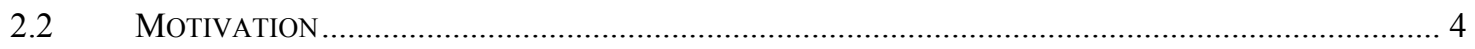

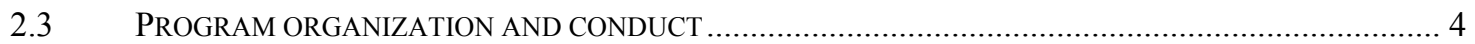

3. MEDICAL DEVICE PROJECTS ................................................................................................. 6

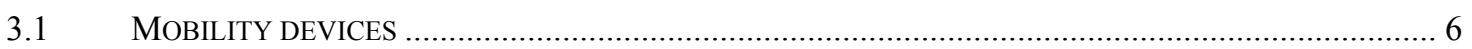

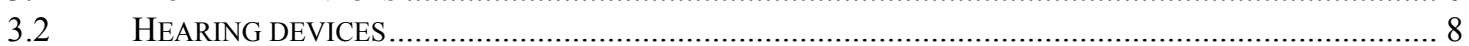

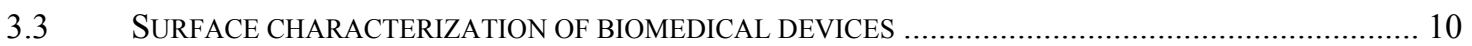

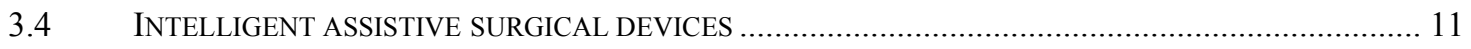

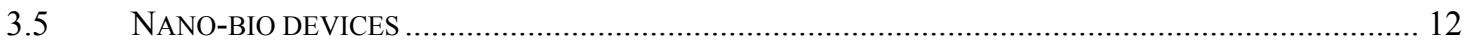

4. HEALTHCARE INFORMATICS PROJECTS................................................................................ 14

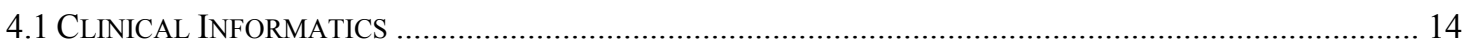

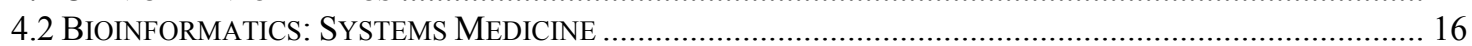

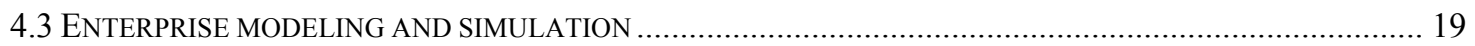

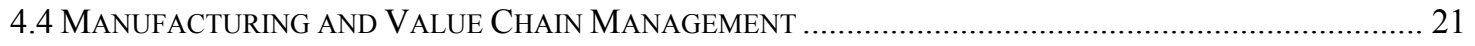

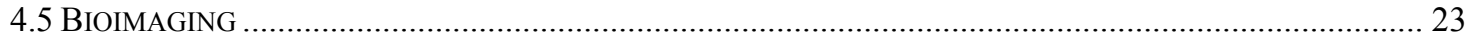

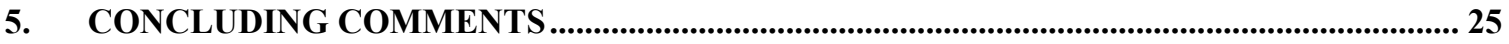

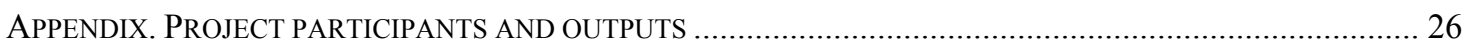

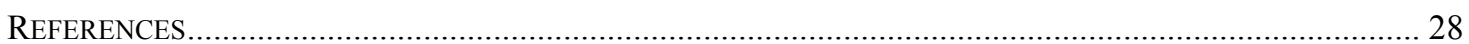

STANDARDS PUBLISHED BY COMMITTEES IN WHICH PROGRAM MEMBERS PARTICIPATED ......................... 30

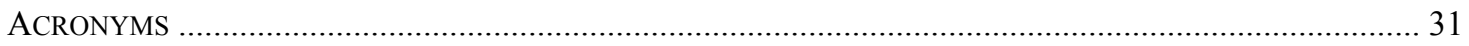




\title{
Manufacturing Metrology and Standards for the Health Care Enterprise Program Summary
}

\author{
Ram D. Sriram and Steven J. Fenves, editors
}

Project leads: Roger V. Bostelman, Nicholas G. Dagalakis, John A. Dagata, Shaw C. Feng, Steven J. Fenves, Charles R. McLean, Victor Nedzelnitsky, Ram D. Sriram, Eswaran Subrahmanian, Theodore V. Vorburger, and Randall Wagner

\begin{abstract}
The Manufacturing Metrology and Standards for the Health Care Enterprise program was initiated in FY2005 as part of the overall reorganization of the Manufacturing Engineering Laboratory (MEL), with Ram D. Sriram as the Program Manager. The goal of the program was stated as follows "to apply proven MEL manufacturing technology and expertise to healthcare systems, biomedical devices and equipment, and biomedical data management." This report summarizes the activities, achievements and impacts of the program.
\end{abstract}

\section{Introduction}

\subsection{Rationale for the report}

The Manufacturing Metrology and Standards for the Health Care Enterprise program was initiated in FY2005 as part of the overall strategic reorganization of the Manufacturing Engineering Laboratory (MEL), with Ram D. Sriram as the Program Manager. It became one of the new exploratory programs in MEL.

Since the program is conducted through a number of largely autonomous research projects, this report provides a comprehensive overview of the program as a whole since the program planning report was submitted in August 2004.

\subsection{Organization of the report}

After a brief overview of the program in Section 2, the individual projects are summarized in Sections 3 and 4. The concluding comments in Section 5 summarize the program as a whole. 


\section{The program}

\subsection{Program goal}

The goal of the program is to apply proven MEL manufacturing technology and expertise to healthcare ${ }^{1}$ systems, biomedical devices and equipment, and biomedical data management.

The program is predicated on the observation that the healthcare and manufacturing industries share many similar organizational, technological and informational aspects. Thus, the healthcare industry as a whole is a customer for the metrology, standard-setting support and technology approaches and solutions that MEL has developed for the manufacturing sector that are transferable or adaptable to the healthcare sector.

The benefit of the program to the healthcare industry is an infrastructure for the development of improved organizational, technological and informational support methodologies for health care delivery. NIST's contributions are intended to enable more effective development and application of biological and medical knowledge to practical problems.

\subsection{Motivation}

The healthcare industry is perennially facing major challenges: increasing costs, unacceptable error rates, and dissatisfied patients and providers. Healthcare costs in the United States were about $14.9 \%$ of the GDP - \$1.6 trillion - in 2002, estimated to be 1.9 trillion in 2005 and projected to rise to 3.6 trillion by 2014. These costs are also a major concern for U.S. industry, as escalating healthcare costs are impeding our ability to globally compete.

It is the expectation of the program that importation, adaptation, and application of technology and expertise from the manufacturing sector can jump-start the applicable aspects of healthcare delivery and the improvement of medical devices.

\subsection{Program organization and conduct}

The program is organized along two dimensions:

1. Medical devices and

2. Healthcare informatics.

Within each of the two dimensions, the program is conducted through a number of largely autonomous research projects. The program manager oversees the project activities and identifies issues of shared interest among projects. The various projects proposed in August 2004 were as follows:

\footnotetext{
${ }^{1}$ We use the words "healthcare" and "health care" interchangeably.
} 
Manufacturing Metrology and Standards for the Health Care Enterprise Program report

\section{Medical devices}

1.1 Mobility devices. Develop test methods and performance metrics, sensor data, standards and specifications necessary for intelligent assistive devices for wheelchair dependents and the blind.

1.2 Hearing devices. Develop test and measurement methods, data, standards and specifications necessary for the characterization, manufacturing, and testing of hearing devices and related diagnostic equipment.

1.3 Surface characterization of biomedical devices. Develop test procedures for characterizing the surfaces of medical devices that relate to device function and failure behavior.

1.4 Intelligent assistive surgical devices (medical robots). Work with an American Standards for Testing of Materials (ASTM) committee, the Food and Drug Administration (FDA), medical robotic research groups and University Hospitals for the establishment of Intelligent Assistive Surgical Device standards. This work will extrapolate on our previous work on industrial robot performance and safety standards, related metrology, instrumentation, and artifact and marker design.

1.5 Nano-bio devices. Develop protocols for high-resolution imaging of individual components and associated complexes of the constituents of nanoparticle drug delivery systems (NDS). Demonstrate imaging of the cell transfection process with fixed and live cells using such systems. Nano scale devices have components with features which range between 1 micrometer and 1 nanometer.

\section{Healthcare informatics}

2.1 Clinical informatics. Extrapolate from MEL's experience in information modeling and research supporting information interchange standards development for the manufacturing industry to provide experience, assistance and leadership for related activities in the health care informatics field.

2.2 Bioinformatics. Adapt and extend NIST's expertise in information modeling, information interchange and standards development in the manufacturing arena to the field of bioinformatics, leading to synergisms with bioinformatics research and practice and consolidation of the bioinformatics knowledge base.

2.3 Enterprise modeling and simulation. Explore the applicability of the modeling and simulation technologies developed in MEL to healthcare systems; explore means for disseminating this information to the shareholders in the healthcare industry.

2.4 Manufacturing and value chain management. Develop interface specifications and interaction protocols for integrating manufacturing and e-commerce software solutions into biomedical device value chain and enhance existing standards for device integration.

An additional project on Bioimaging, funded as part of the American Competitive Initiative (ACI), was added to the Healthcare informatics portfolio. The goals of this project are: 
1) develop standardized representation schemes and archival techniques for a webbased data storage system that will enable semantic annotation, content-based search and retrieval, visual browsing, cross linking between pathological states and image features, and appropriate levels of security, and

2) develop an ontology to represent the semantics of archived images that can be used as the basis for open standard implementations of archive systems.

\section{Medical device projects}

\subsection{Mobility devices}

\section{Introduction and goals}

Mobility must be viewed as being essential to the outcome of the rehabilitation process of wheelchair-dependent persons and to their successful (re-)integration into society. Just as important are the lift devices that lift patients into wheelchairs and other seats. In 2005, NIST ISD (Intelligent Systems Division) began the Healthcare Mobility Project to target patient lift and mobility. ISD researchers looked at currently available technology through a survey of patient lift and mobility devices. The survey showed that there is a need for technology that (a) includes mobility devices that can lift and maneuver patients to other seats and (b) can provide for rehabilitation to help the patient become independent of the wheelchair. The survey also noted that there are no standards nor performance metrics for either technology.

\section{Technical overview:}

Six major activities were undertaken:

1) HLPR chair concept. NIST designed an HLPR (Home Lift, Position, and Rehab) Chair testbed to investigate patient transfer including specific areas of mobility, lift and rehabilitation toward safety standards, performance measurements of such devices, and advanced autonomous controls. The HLPR Chair testbeds are based on a manual, steel, inexpensive, off-the-shelf, and sturdy forklift. The forklift includes a $\mathrm{U}$-frame base with casters in the front and rear and a rectangular vertical frame. The lift and chair frame is small enough to pass through even the smallest residential bathroom doors.

2) Seat/stand. The patient seat/stand mechanism is a double, nested and inverted Lshape where the outer $\mathrm{L}$ is a seat base frame that provides a lift and rotation point for the inner L seat frame. The $\mathrm{L}$ frames are made of square, aluminum tubing. The outer $\mathrm{L}$ is bolted to the lift device while the inner $\mathrm{L}$ rotates with respect to the seat base frame at the end of the L. Drive and steering motors, batteries and control electronics along with their aluminum support frame provide counterweight for the patient to rotate beyond the wheelbase. When not rotated, the center of gravity remains near the middle of the HLPR Chair. When rotated to $180^{\circ}$ with a $136 \mathrm{~kg}$ $(300 \mathrm{lb})$ patient on board, the center of gravity remains within the wheelbase for safe seat access. Heavier patients would require additional counterweight. 
3) Propulsion. The HLPR Chair is powered similarly to typical powered chairs on the market. However, the HLPR Chair has a tricycle design to simplify the need to provide steering and drive linkages and to provide for a more vertical and compact drive system design. The front of the robot has two casters mounted to a U-shaped frame. Steering is a novel single wheel design, hard stopping the wheel at just beyond $180^{\circ}$ for safety.

4) Patient placement on other seats. Healthcare providers explained that caregivers placing patients on toilets provide the worst condition for injuries to caregivers because of confined spaces. To place an HLPR Chair user on another seat, they can drive to it. Once there, the HLPR Chair rotates the footrest up and beneath the seat and the patient's feet are placed on the floor personally or by a caregiver. The HLPR Chair inner L-frame can then be rotated manually with respect to the chair frame allowing the patient to be above the toilet. Padded torso lifts then lift the patient from beneath his/her arm joints similar to crutches. The seat, with the footrest beneath, then rotates from horizontal to vertical behind the patients back clearing the area beneath the patient to be placed on the other seat.

5) Patient lift. Patient lift is designed into the HLPR Chair to allow user access to high shelves or other tall objects while seated. The HLPR Chair's patient lift is approximately $1 \mathrm{~m}$ (36 in) to reach what a typical standing $2 \mathrm{~m}(6 \mathrm{ft})$ tall person could reach. The additional height comes at no additional cost of frame and only minimally for actuator cost. Lift is achieved by a $227 \mathrm{~kg}(500 \mathrm{lb})$ max. lift actuator that can support $681(1500 \mathrm{lb})$ statically. The actuator can be replaced with a higher capacity unit if needed. The actuator connects to a lift plate with a steel chain that is fixmounted at one end to the HLPR Chair frame and to the lift plate at the other end.

6) Patient rehabilitation. HLPR Chair enhances patient rehabilitation through a load sensor and control on the lift actuator. The researchers designed rehabilitation into the HLPR Chair to allow, for example, stroke patients to keep their legs active without supporting the entire load of the patient's body weight. The patient, once lifted, could walk while supported by the HLPR Chair driving at a slow walking pace towards regaining leg control and eliminating the need for a wheelchair.

For further details see http://www.isd.mel.nist.gov/healthcaremobility/.

\section{Achievements}

Two HLPR chairs have been built: (a) the first to study the advanced autonomous control and transfer to the University of Pittsburgh for collaboration on future standards, and (b) the second to study manufacturability, ergonomics. The latter was transferred to the Florida Gulf Coast University for collaboration on future interoperable controls and to study static and dynamic stability performance testing.

Achievements also included:

1) Five published papers

2) Two NIST Internal Reports

3) Several presentations

4) Workshop presentation at ICDR 07 in Arlington, VA 
5) Meetings with Walter Reed Army Medical Center, University of Pittsburgh and Florida Gulf Coast University

6) Two videos of the HLPR Chair

7) HLPR Chair Tech Beat Article which provided over 1000 initial Google hits

8) Application for patent on "Modifications to the HLPR Chair." The HLPR Chair 1 is in the public domain.

\section{Impacts}

The HLPR Chair impacts healthcare service providers, reducing the need for manual labor in transferring patients. In this field 1 in 3 nurses becomes injured from patient transfer, a rate that is about $21 / 2$ times higher than that of all other general industries. Injuries in Nursing Homes alone this year will reach 200,000 incidents at a cost of almost $\$ 1$ billion.

\subsection{Hearing devices}

\section{Introduction and goals}

Hearing loss afflicts approximately 30 million Americans of all age groups. The Better Hearing Institute (2005) quantified that the impact of untreated hearing loss is "in excess of $\$ 100$ billion annually" for America's 24 million hearing-impaired who do not use hearing instruments, and that the corresponding annual "cost to society could be well in excess of $\$ 18$ billion due to unrealized taxes." The diagnosis and treatment of hearing loss and the currently excessive incidence of failures of hearing devices must be improved: nearly one of every five aids dispensed in 2000 was returned to the manufacturer for credit, totaling $\$ 500$ million in 2000 .

Auditory diagnostic and prosthetic devices include a wide variety of analog instruments and programmable digital devices that contain analog components and are capable of digital signal processing. The greatly enhanced features and capabilities of evolving devices call for suitable methods and standards enabling performance specification, replicable measurements, production testing, and quality assurance.

The goal of this project is to develop test and measurement methods, data, standards and specifications necessary for the characterization, manufacturing, and testing of hearing devices and related diagnostic equipment so as to help designers, diagnosticians, and dispensers to control the very large societal costs of untreated hearing loss as well as the excessive incidence of failures in patient outcomes.

\section{Technical Overview}

Hearing devices include hearing aids, cochlear and brainstem implants, and related diagnostic equipment. Product design, production quality control, and acceptance testing/conformity assessment require well-defined and replicable measurement procedures. Often, a given type of device is characterized by many parameters that must be adjusted to meet the needs of individual patients. Proper determinations of these individual needs, as well as the verifications of parameter selections, adjustments, and patient outcomes, often require complicated diagnostic equipment and procedures 
designed and optimized for that type of device. Lack of compatibility of devices, equipment, or procedures from different manufacturers can be an important issue.

The Department of Veterans Affairs (VA) needs NIST to characterize hearing aids that the VA submits. Hearing aid characteristics are determined by means of measurements conducted in accordance with the criteria and procedures established or approved by the VA. In addition, the VA needs NIST to make any necessary alterations in test methods based on new developments and designs in the field of hearing aids and related diagnostic equipment and techniques. These needs are met by Interagency Agreements between the VA and NIST. The technical approach applies the experience, specialized laboratory facilities and equipment of NIST to the task. Personnel at NIST participate in national and international standardization activities in this area through the American National Standards Institute (ANSI) and the International Electrotechnical Commission (IEC), which leverage the effect of the NIST work. Specialized laboratory facilities and equipment include anechoic chambers, acoustical measurement instrumentation including custom-designed systems and computer programs, and anthropometric manikins designed for hearing aid and related acoustic research.

\section{Achievements}

Achievements included:

1) Completed directivity index measurements on two directional hearing aids which were circulated among several laboratories involved in a directional measurement round-robin organized within the ANSI Hearing Aid Working Group S3/WG48.

2) Performed special testing on audio headsets designed to meet the requirements of The National Library Service for the Blind and Physically Handicapped (NLS).

3) Received an A rating (numerical score of 100) in every category included on the NIST Customer Satisfaction Report Card completed by the VA.

4) Developed test procedures for measuring the insertion gain of open-fit hearing aids.

5) Developed preliminary plans with the VA to conduct tests of open ear and fullysealed deep-fitting postauricular hearing aids.

6) Performed measurements using vented earmold and E-A-R ${ }^{\circ}$ plugs in the occlusion effect simulator with the system in its current configuration.

7) Developed plans to acquire occlusion effect data with vented earmolds as a function of vent size for comparison with data reported from a clinical study that examined the magnitude of the occlusion effect as a function of vent size for real ears.

8) Worked with an audiologist at the Washington, DC VA Medical Center to produce ear impressions required to fabricate earmolds for testing in the occlusion effect simulator.

9) Participated in the publication of ANSI S3.35-2004 Method of Measurement of Performance Characteristics of Hearing Aids Under Simulated Real-Ear Working Conditions.

10) Participated in meetings of the ANSI Hearing Aids Working Group S3/WG48.

11) As Technical Advisor to the U.S. National Committee of the International Electrotechnical Commission (USNC/IEC) for IEC TC 29, Electroacoustics, provided recommendations to the USNC/IEC and participated in the publication of seven international standards. 
12) Provided the recommended USNC/IEC positions and comments on fourteen draft IEC standards on hearing devices and related equipment.

13) Provided comments and votes on six ANSI draft standards and documents.

\section{Impacts}

Services performed by NIST for the VA play a key role in helping VA officials make purchasing decisions regarding hearing aids, including the way in which the VA specifies the types of aids it purchases and the characteristics of these aids. These services affect an even larger population than veterans with hearing impairment, since the VA has a major effect on the way in which technology and services are provided to all persons in the U.S. with hearing-related problems. According to The Hearing Journal (February 2001), the audiology program of the VA is "far reaching in its goals and has a strong ripple effect throughout the whole U.S. hearing healthcare community."

The development of measurement techniques and recognized, widely accepted standards for characterizing hearing devices is supporting improved production and quality control of these devices, as well as improved selection and adjustment of these devices to meet the diverse needs of individual patients.

\subsection{Surface characterization of biomedical devices}

\section{Introduction and goals}

We aim to develop test procedures for characterizing the surfaces of biomedical devices that relate to device function and failure behavior. The function of medical devices is critically dependent on their surface topography. These devices include transcutaneous pretreatment devices, orthopedic implants, heart pacemaker electrodes, dental preparations, and intravascular stents. In addition, the properties of test cultures may be strongly correlated with their surface topography.

\section{Technical overview}

We are investigating the importance of surface roughness and geometry for two applications:

1) The correlation of surface roughness of vascular smooth muscle cells to their response under macroscopic strain.

2) The effect of surface topography on the function of transcutaneous pretreatment devices.

Optical confocal microscopy is being tested as the surface roughness measurement tool.

\section{Achievements}

The work emphasized the first direction described above. In collaboration with the Polymers Division in the NIST Material Science and Engineering Laboratory (MSEL), we studied the roughness of extracellular matrix proteins coated onto substrates of deformable polydimethylsiloxane elastomer modified by different surface treatment methods. Results from physically adsorbed, chemically attached, and covalently bonded proteins were compared to determine the best surface treatment for high coverage and 
uniformity. Surface topography images and profiles were obtained using a NanoFocus $\mu$ Surf confocal microscope with a rotating Nipkow disk and a xenon light source over a field of view of $800 \mu \mathrm{m} \times 800 \mu \mathrm{m}$ with an $\mathrm{x}$ and y pixel resolution of $1.56 \mu \mathrm{m}$.

A number of alternative surface treatments were investigated. We note the results for two of these. A treatment of silane followed by glutaraldehyde produced protein surfaces with higher surface roughness $(\approx 32 \mathrm{~nm})$ and lower protein coverage than the others and therefore this treatment seemed less effective than the others. By comparison, the pretreatment of silane incubated at $70^{\circ} \mathrm{C}$ produced high protein coverage and high uniformity along with modest surface roughness $(\approx 10 \mathrm{~nm})$. This type of treatment will be explored more fully in subsequent tests on smooth muscle cells in future studies involving mechanical stress of the protein coatings. The surface roughness measurements were useful for characterizing the quality of the protein structures.

\section{Impacts}

The Precision Engineering Division's confocal microscope contributed successfully to the assessment of the surface quality of protein coatings and will be effective in future studies of these systems in collaboration with bio-engineering researchers.

\subsection{Intelligent assistive surgical devices}

\section{Introduction and goals}

Approximately one million arthroplasty (joint reconstruction) operations are performed per year throughout the world. Sources indicate that $8.8 \%$ of revision joint surgery could be attributed to malpositioning of the implant. Computer Assisted Orthopaedic Surgery (CAOS) systems help reduce the variability of the positioning of prosthetic components. The project objective is to work with representatives of the users and manufacturers of these systems and tools in order to develop standards and tests which can help minimize surgical problems and achieve optimum performance results.

\section{Technical overview}

CAOS systems help reduce the variability of the positioning of prosthetic components, thus permitting a more consistent placement of the prosthetic components. CAOS systems consist of tracking sensors, tracking markers, a computer and other electronics. During an operation the markers are attached to bones, surgical tools and implants. The position of the markers is determined with respect to a reference frame and based on that information the position and orientation of tools, bones and implants is calculated.

We have developed a simple, lightweight, easy to use operating room high precision artifact, called phantom by medical professionals, which can be used at the beginning of each operation for a quick calibration and performance test of most CAOS systems. A longer and more thorough calibration and performance test can be performed early in the morning before the first surgical operation. 


\section{Achievements}

Achievements included:

1) Conducted a Medical Devices Metrology and Standards Needs workshop.

2) Submitted for review five USMS Medical Devices Metrology and Standards Needs.

3) Completed the machining of the Horizontal Joint-Computer Assisted Orthopaedic Hip Surgery (HJ-CAOHS) artifact. This artifact is using a commercially available precision magnetic ball and socket to simulate the human hip joint. The phantom is made of a femur bar, a magnetic ball and socket and an L shape XY coordinate frame.

4) Completed the calibration of the HJ-CAOHS Phantom.

5) Published two papers.

\section{Impacts}

The workshop on "Medical Devices Metrology and Standard Needs," was attended by a large number of medical professionals and medical devices suppliers and researchers. Acting as a catalyst, the workshop has created potential collaborations among the participants and has attracted a lot of attention by NIST, FDA and NIH researchers. For further details see http://www.isd.mel.nist.gov/medical_devices/.

Adoption of the NIST CAOS phantoms has the potential to significantly reduce the number of malpositioning failures of arthroplasty surgical operations. This will reduce the suffering of hundreds of thousands of patients and save tens of millions of dollars annually.

\subsection{Nano-bio devices}

\section{Introduction and goals}

Nanoparticle delivery systems (NDS) -- multicomponent, multifunctional vehicles for targeted delivery of diagnostics and therapeutics -- represent one of the most promising developments in nano-medicine. Imaging as physical measurement is expected to play a crucial role in future drug discovery and development processes. However, the technology for characterizing nano-bioparticles, biologically functional and organized structures in the size range of $10-200 \mathrm{~nm}$, is still in its infancy. The National Cancer Institute (NCI) and Food \& Drug Administration (FDA) are seeking to replace slow and expensive biological and biochemical assays. NCI's Nanotechnology Characterization Laboratory's (NCL) "characterization cascade" is intended to qualify NDS prior to FDA approval, but there are no structure-function relationships that currently link individual physical measurements to in-vitro and in-vivo biological properties. In collaboration with Georgetown University Medical Center (GUMC) and the University of Akron, we have developed a systematic methodology for directly correlating physical and biological information about NDS from imaging data. This approach utilizes fluid scanning probe microscopy (SPM) as a versatile tool for high-resolution imaging of, and obtaining dynamical information about, functional biological systems in solution. Fluid SPM refers to the operation of a scanning probe microscope under solution conditions. It includes the development of specialized techniques for sample preparation, stable feedback operation of an oscillating probe tip in solution, and quantitative force measurements of chemical, 
mechanical, electrical, hydrodynamic, and magnetic interactions of the molecular constituents of an NDS. Appropriate preparation and measurement techniques for physical characterization of nano-bioparticles have been developed in parallel with invitro and in-vivo GUMC efficacy studies in order to facilitate drug discovery and development.

\section{Technical overview}

Two major activities were undertaken:

1) Fabrication of standard phantoms for magnetic resonance imaging. Optimal design and formulation of a nanoparticle delivery system for biomedical applications requires development of a systematic methodology for directly correlating physical and biological information through high-resolution imaging of functional biological entities. We have achieved our goal of calibrating spatial resolution and threshold detection limits for iron-oxide and gadolinium-containing Magnetic resonance imaging (MRI) contrast agents and have validated the nanoscale size dependence of GUMC formulations based upon magnetic measurements at NIST.

2) Advanced imaging for targeted nanoparticle delivery systems (NDS). Fluid scanning probe microscopy uniquely satisfies the needs for high-resolution imaging of dynamical biological systems in solution. We have achieved our goal of providing an essential linkage between biomedical and physical disciplines using functional NDS by successfully performing size, size distribution, particle aggregation, and physical activity on systems with encapsulated gene therapy, chemotherapy, and magnetic resonance imaging contrast agents.

\section{Achievements}

Achievements included:

1) Demonstrated optimized methods for immobilizing intact liposome-based nanoparticle delivery systems (NDS) for scanning probe microscope (SPM) imaging and characterization under fluid conditions:

- Optimized mica, poly-L-lysine (PLL)-mica, and silane-modified silicon substrate preparation

- Demonstrated UV/ozone tuning of surface potential of PLL-mica substrates

- Developed surface-potential patterning techniques for PLL-mica substrates

- Evaluated rotating disk method for surface potential determination of substrates

- NDS compressibility

2) Fabricated and evaluated improved magnetic coatings for fluid and dry magnetic force microscopy (MFM) for characterizing dispersed and aggregated MRI contrast enhancement agents:

- Quantitative methods for establishing iron concentration and particle density of NDS-encapsulated SPIOs

- Magnetic characterization of GUMC SPIOs (MFM, SQUID, MRI)

3) Investigated quantitative particle size analysis of gold reference nanoparticles, NDS and SPIOs from SPM images and comparison with dynamic light scattering (DLS): 
- Lift-mode techniques for validating intactness and measuring compressibility of adsorbed liposomes

- Quantitative particle size analysis from SPM images and inter-comparison with DLS

4) Participated as the NIST representative on the organizing committee of a National Science Foundation-sponsored workshop on Nano-Bio-manufacturing, March/April 2008.

\section{Impacts}

1) The rotating disk method for measuring surface zeta potentials of substrates as a critical step in optimizing the attachment of liposome-based NDS will enable highmagnification imaging techniques to perform quality assurance and potency assessment of nanoparticle delivery systems at both individual and bulk particle level during drug development and optimization.

2) Correlation of size, aggregation, and functionality of magnetic nanoparticles as magnetic resonance imaging contrast agents will hasten the implementation of their diagnostic use and improve early detection of disease.

3) Fluid SPM imaging provides a unique means of validating statistical size distributions obtained from dynamic light scattering and those obtained by electron microscopy, thus reducing reporting discrepancies that arise during the drug approval process.

4) The standardization and measurement work enables repeatable and reproducible measurement of physical properties of nanoparticle delivery systems and their components. This will help accelerate drug discovery and development.

\section{Healthcare informatics projects}

\subsection{Clinical Informatics}

\section{Introduction and goals}

The goal of the project is to extrapolate from MEL's experience in information interchange standards development to provide assistance for related activities in the healthcare informatics field. 


\section{Technical overview}

Three major activities were undertaken.

1) Survey of opportunities. As initially proposed, an extensive survey of the strategic focus area in healthcare informatics was completed. The report presents three aspects of clinical informatics of most immediate interest:

- Approaches and standards for the integration and interchange of health information

- Controlled vocabularies of concepts that cover medical knowledge and

- Evidence-based medicine (EBM), filtering and disseminating healthcare knowledge.

2) Assistance to the Office of the National Coordinator for Health Information Technology (ONC). The Architecture Development Facilitator (ADF) was developed to assist the Office of Network Coordinator for Health Information Technology of the Department of Health and Human Services (HHS) and its agents in the deployment of the Nationwide Health Information Network (NHIN). ADF was intended to serve as the repository of a set of predefined compatible health care IT components, called artifacts, which can then be combined to design, configure and deploy a large variety of health care IT system architectures.

3) Analysis of workflow in physicians' offices. The goal of this study is to understand and map detailed workflow for small physician offices. The study is to be performed with in-situ interviews and use of questionnaires. Our goal to confirm and elaborate some of the findings about use and adoption of EHR and other automation in small physician offices.

\section{Achievements}

Achievements included:

1) The strategic focus survey served as both an inventory of capabilities and a plan for action. The ONC project on the ADF was a direct outgrowth of both the contacts made between MEL and ITL and of the capabilities described in the report.

2) The design of ADF responded to the evolving nature of the NHIN envisaged by ONC. It made absolutely no assumptions about the upper levels of the NHIN architecture and did not impose any constraints on any architecture that may evolve, from the most highly centralized one to the fully decentralized one. ONC chose to redirect its efforts towards a network of networks, which made the repository of small, atomic components redundant.

3) A questionnaire was developed and submitted to the Department of Commerce for required clearances. A report on the workflow and sources of delay in processing patients and documents will be written after we get the required clearances.

\section{Impacts}

1) The impact of the strategic focus survey has been largely internal to NIST by identifying compatible interests and specific focus areas to pursue. 
2) While the directions of $\mathrm{ONC}$ and the ADF project paralleled each other, the ADF team had multiple opportunities to impact a number of ONC initiatives beyond the ADF proper.

3) The main impact of the workflow studies of small offices will be on the identification of benefits and causes for EHR implementations in small physician offices and the need for an information network infrastructure to facilitate their integration.

\subsection{Bioinformatics: Systems Medicine}

\section{Introduction and goals}

The Human Genome project has provided us with a map of the human genome, the study of which is called genomics. This has led to developments of a wide variety of technologies for profiling a person's DNA. It is predicted that we will be able to provide the complete genetic profile of a person in a cost effective manner in the near future. Genomics, along with the evolving fields of proteomics - which involves the study of the functions of all expressed proteins and protein networks - and metabolomics - which involves the rapid, high throughput characterization of the small molecule metabolites found in an organism - is changing the practice of medicine.

The integration of various "omics" to understand a biological system is the field of systems biology (SB). Essentially, systems biology involves gathering comprehensive sets of data that define and quantify the elements of a particular biological system and computationally analyze them to establish functional and dynamic connections. The premise is that no molecule in biology acts alone. Systems biology in a clinical context is called systems medicine (SM). We will use the term SB\&M to denote systems biology and medicine. As Leroy Hood, a member of NIST's VCAT, put it, "Systems approaches to medicine are transforming current diagnostic and therapeutic approaches to medicine, and together with new technologies, will enable a predictive and preventive medicine that will lead to personalized medicine."

\section{Technical overview}

Biological systems are characterized by multiple interlinked pathways of interaction at various levels. These levels span the molecular level to the cellular level and to the multi-cellular organism level. The interactions within and across these levels are critical to the fundamental understanding of SB. The goal of SB is to formalize descriptions of these processes and interactions. The state of art in SB and absence of a grand theory open the space of exploration to extending the traditional systems engineering (SE) principles to better understand the challenges of SB. This work is directed at a survey of accomplishments in SB and exploring the feasibility of applying SE approaches to this emerging field. The approach will be based on feasibility of applications of current computational formalisms available is SE, their limitations, and the requirements for extending these formalisms for developing a deeper understanding of biological functions.

Three major activities were undertaken:

1) Identification of proteins involved in diabetes condition change after stomach stapling. The Hershey Medical Center (HMC) recently discovered that some 
morbidly obese patients, who were initially diabetic, found themselves free of diabetes after stomach stapling surgery. HMC was interested in understanding the mechanism involved in this process. Differential Protein Expression Profiling, which uses mass spectrometry, makes it possible to get a "snapshot" of the biological state of the patient before and after the surgery in terms of relative protein levels. Using this information and various statistical methods and wavelet analysis we can generate a protein-protein interaction network that can be used to determine the pathways involved in the curing of diabetes.

2) Evaluating protein-protein interaction software. The primary goal of this project is to develop metrics for evaluating protein-protein interaction software. We ventured into this by selecting a problem and then evaluating various tools for this problem. The problem we selected involves the interaction of two proteins involved in Alzheimer's disease (AD): Cdk5 and p25. Cdk5 (cyclic dependent kinase 5) plays a significant role in Alzheimer's disease (AD) and other neurodegenerative diseases; Cdk5 was discovered at the National Institutes of Health, while studying the regulation of neuronal cytoskeletral proteins by phosphorylation. Essentially, Cdk5 phosphorylates another protein tau at AD-specific phospho-eptiopes when it associates with a protein p25. p25 is a truncated activator, which is produced from another protein $\mathrm{p} 35$ - a neuron specific cdk5 activator-when exposed to amyloid beta peptides. However, another peptide called CIP (Cdk5 Inhibitor Protein) will make the Cdk5/p25 combination inactive. Our goal is to better understand the role of geometry in the interactions between $\mathrm{Cdk} 5, \mathrm{p} 25$, and tau, so as to develop metrics for evaluating various docking programs. To do this we first obtained the various trajectories of conformational transition for Cdk5, p25 and CIP and then we defined a procedure to perform a preliminary comparison of the relative abilities of p25 and CIP to conform to the kinase. Our procedure compares the geometric affinity of p25 and CIP with Cdk5 for each given conformation. It consists of aligning a given Cdk5 conformation with the corresponding p25 or CIP conformation in compliance with the conservation hypothesis. This means that Cdk5 and CIP or p25 should be aligned such that their interface interaction is as close as possible to $\mathrm{Cdk} 5 / \mathrm{p} 25$ active conformation. A distance function is finally evaluated after the alignment, and it yields the geometric affinity between CIP or p25 with Cdk5.

3) Extracting protein-protein interaction sentences from literature. Protein-Protein Interaction (PPI) information is critical to understanding the function of individual proteins and the organization of entire biological processes. Therefore, current biomedical literature is replete with articles that describe PPI experiments, which specify individual interaction proteins and the corresponding interaction types. Protein interaction databases also have been developed by utilizing these biomedical articles. However, the rapid growth of the literature makes it difficult to manually find the necessary information. In addition, the dynamic nature of biology makes building the ontology or the database more difficult. We undertook the development of a machine learning-based framework called PIE (Protein Interaction information Extraction system), which identifies PPI information automatically from the biomedical literature. PIE consists of two procedures: article filter and sentence filter. In the article filter, documents are roughly classified to reduce the overhead of the second procedure. The AdaCost algorithm with naive Bayes classifiers is used for the 
article filter, and the SVM classifier with tree kernels is used to identify PPI-relevant sentences for the sentence filter.

\section{Achievements}

Achievements included:

1) Evaluating protein-protein interaction software. We determined theoretically based trajectories of Cdk5, p25 and CIP conformational transitions through FIRST/FRODA technique and geometry-based algorithms implemented in Matlab. Then, based on the obtained Cdk5, p25 and CIP trajectories, we defined a procedure to perform a preliminary comparison of the relative abilities of p25 and CIP to conform to the kinase. In the late stages of the activation trajectory our preliminary comparisons indicate -consistent with our hypothesis - that CIP is more able than p25 to conform to the kinase. Therefore it can be inferred that CIP is less rigid than $\mathrm{p} 25$.

2) Extracting protein-protein interaction sentences from literature. The experimental results confirm that the PPI articles are successfully classified using word-based probability distributions. According to the sentence-level experiments, it is shown that the PPI sentences have their own grammatical structures, and can be represented by a general full parser even without tuning for the PPI domain. The structural patterns are successfully classified using support vector machines with tree kernels. In contrast to the previous rule-based approaches, the machine learning approaches can discover new patterns which are not captured in the known trigger word list. And their automatic way can reduce human efforts and database development cost because the biological databases frequently add or modify new information. These techniques can also incorporate corpus statistics to achieve good performance.

3) Identification of proteins involved in diabetes condition change after stomach stapling. Pennsylvania State University, with a support grant from us, obtained the required data from the Hershey Medical Center. The following tasks were undertaken: 1) Identified the required peptide ratios; 2) Grouped the ratios with respect to range (different ratio ranges); 3) Clustered the different proteins in a particular range; 4) Compared the clusters across all the patients; 5) Identified the significant protein from the cluster properties; and 6) Applied associative rule mining algorithm to identify the other significant proteins.

\section{Impacts}

SB\&M is an emerging field and its impact on the pharmaceutical industry and medical practice will be significant. Interoperability and testing concerns have been repeatedly voiced in several NIH and NIST workshops. Integration of genomics, proteomics, and clinical medicine will lead to innovation in drug discovery and health care delivery, and will result in higher health care quality and lower costs. This will result in significant savings for the $\$ 1.7$ trillion dollar health care industry. We believe that we have made a first step toward achieving these benefits. 


\subsection{Enterprise modeling and simulation}

\section{Introduction and goals}

Simulation, modeling, and game technology can be used to support a broad range of applications pertaining to the health care industry. Our first objective was to conduct a background study to identify needs, issues, and tools with respect to health care simulation. Our second objective was to hold a conference to bring together simulation users from the healthcare industry, academia, and government that have an interest in healthcare simulation standards.

\section{Technical overview}

Principal users of healthcare simulations and standards include healthcare facilities, simulation software vendors, and researchers that will need new interface standards to help develop healthcare simulations or use them to improve facilities and operations.

A detailed background study is currently under review for publication as a NIST technical report. The focus has been to identify healthcare simulation applications. A brief summary of the study follows.

Both discrete-event simulation and system dynamics models are recommended for application at team, organization, and environment levels, leaving out only the individual patient level. Discrete event and system dynamics simulations are recommended for evaluations in health care when interactions between individuals are important for the system aspect being studied.

Simulation tools for application to health care have a higher potential of adoption by health care professionals than other systems analysis tools that may be seen as black boxes. The methodology of simulation tools can be explained much more easily compared to say, mathematical programming. The ability to communicate the simulation methodology is further helped by available visualization facilities with most simulation tools.

Recent literature on simulation applications in health care are surveyed in the study. Emphasis is placed on recent literature and efforts that have not been identified in other reviews of the field.

A classification of types of simulations that are included in the review is described below. Other sections of the report review the literature for successive relevant simulation types. The individual simulation applications are followed by a discussion of some related developments such as integrated simulations and simulation infrastructure issues. The concluding section identifies emerging directions for simulation applications in health care. The relevant simulation techniques for the field of health care are listed below.

1) Computer simulations include models that represent the behavior of a physical system using computer programs that allow limited, if any, interaction during execution. The following types are included: 
- System dynamics simulation represents a system using causal relationships that determine system behavior over time using differential equations.

- Discrete event simulation representation uses events that make step changes in the status of the system over time.

- Agent-based simulation uses representations of behavior of independent entities forming the system to determine the status of the system over time.

- Monte Carlo simulation uses random distributions to represent the outcomes of a system without modeling the passage of time.

- Continuous simulation uses differential equations to represent the physical phenomenon being studied over time.

2) Simulators and simulated environments are built with the objective of training and in some cases assessing clinical skills. This category includes the following types:

- Virtual reality and gaming use highly interactive three-dimensional computer-based graphics and human interaction devices to allow trainees to practice clinical skills.

- Patient simulator devices include the use of mannequins that represent the behavior of full or part of a human body under various conditions.

- Simulated environments include the use of hospital rooms designed to represent real functional rooms such as patient visit rooms with humans role playing as patients or operation theatres with mannequins as the patient.

\section{Achievements}

Achievements included:

1) Reports and Presentations. Prepared two draft journal papers, an in-depth survey presentation (150 slides) and a presentation paper.

2) Conferences and Workshops. Held three workshops and a seminar; participated in five conferences; chaired a session on health care simulation cases; organized two conference sessions; attended meetings at the US Dept of Health and Human Services Headquarters and at Johns Hopkins University Medical School; participated in the Nurse Work Environment Innovation Summit and the TATRC $7^{\text {th }}$ annual Advanced Medical Technology Review.

3) Prototype Simulations and Models. Developed a discrete event simulation model of a hospital emergency room in Promodel-Medmodel; collaborated with Dartmouth College on the Triage training software; and provided 3D Scanning support for the Surgical Devices project reported in Section 3.4.

\section{Impacts}

The most important impact of the project has been in educating the public on the need for standards to enable the more widespread use of simulation in the healthcare industry. There has been significant interest in a NIST-led activity to help establish data standards that would enable healthcare facilities to model and simulate their organizations. 


\subsection{Manufacturing and Value Chain Management}

\section{Introduction and goals}

The worldwide revenue in the biomedical device and equipment market was $\$ 176.2$ billion in 2003 and was expected to grow $6.5 \%$ over the next three years. The medical device industry employs over 310,000 people. Approximately $\$ 62$ billion worth of goods a year is traded. In 1996, a study entitled Efficiency Healthcare Consumer Response concluded that $\$ 11$ billion was wasted annually because of healthcare supply chain inefficiency, such as inadequate quantities being shipped, supplies not reaching their destination, etc. Furthermore, competition and global economic pressures have led to consolidation in the medical equipment vendor community and the use of manufacturing outsourcing as a cost-containment measure.

The goal of the project is to support the biomedical device industry in developing critical information and communication standards that will enable integrating and optimizing the value chain performance, ensuring product quality and safety, and providing the traceability of a medical device and its components throughout the device life cycle. Specifically, we participated in relevant standard development organizations to develop an XML schema of medical device manufacturing and e-commerce using semantic-based information modeling methods.

\section{Technical overview}

We conducted a workshop to acquire industry and research needs on standards in manufacturing and value chain management in healthcare. Using this workshop results we plan to aid in the development of needed standards for supply chain management for medical and pharmaceutical products. The standards will enable robust e-business transactions and tracing and tracking of timely delivery. In the standard development process, available text-based e-commerce standards, ANSI, and ISO/IEC standards will be harmonized with the new standards. The standards will be tested at NIST and implemented by the partnering companies. Types of standard include business transaction, auto-identification, product specification (for regulation and auditing), process documentation and notification to FDA for regulation and auditing, and quality standards (for regulation and auditing). Tasks to complete these standards include: standards development, standards harmonization, conformance testing, semantic models, interaction protocol, and product/process identification repository service. Infrastructure research includes: dynamic representation and implementation of services, information models, interactions, terminologies, and business rules. A workshop is to be held to identify industry needs.

\section{Achievements}

Achievements included:

1) Surveyed industry needs on medical value chain management. The Institute of Medicine (IOM) reports medical errors result in approximately $\$ 29$ billion in excess healthcare expenditures and lost productivity each year. The Efficient Healthcare 
Consumer Response (EHCR), a broad-based industry effort to promote efficient practice in the healthcare supply chain, estimated there to be $\$ 11$ billion in attainable savings annually by reducing medical errors with appropriate standards for using currently available information technology. Various other reports from the Medical Device and Diagnostic Industry (MDDI) also reports more than $\$ 6$ billion can be save with appropriate information standards and technology to better buy products, track delivery, and monitor the use of medical devices in hospitals and clinics.

2) Attended the Medical Design and Manufacturing Expositions. Interacted with stent, catheter, surgical tool, artificial hip/joint, diagnostic tool, and endoscope manufacturing company representatives.

3) Conducted a symposium to study industry needs. The symposium was held in November 2006 in Minneapolis, organized with the University of Minnesota. Over 100 persons attended from major medical device companies, their suppliers, governments, and universities. The symposium provided an open forum for industry, government, health providers, and academia to discuss technological and standards issues. Information and communication standards were identified as a critical component in the medical device industry for supporting supply chain optimization, tracing and tracking expensive medical devices, and ensuring product quality and safety.

4) Contributed to the HIBCC (Health Industry Business Communications Council) in the development of a draft XML-based specification on selected e-business transaction messages commonly used in supply chains. Drafted XML schema documents on invoices and orders to support the HIBCC e-Business standards for healthcare supply chains. The XML schemas provide internet-based e-Business transactions that will free companies from transaction fees using the Value Added Network. Also, the World Wide Web Consortium (W3C) markup language provides defining interaction protocols and service descriptions and announcements. We also conducted a literature study on healthcare supply chain management. The standard suites studied include ANSI X.12, UN/EDIFACT, ebXML, and UBL.

\section{Impacts}

1) Expedited HIBCC's transition from using EDI standard to XML-based standard. Since XML-based e-business standards are central to solve healthcare efficiency problems, the NIST involvement helped save heath care costs. Member HIBCC companies include John and John Healthcare, Cardinal Health, BD, Hospira, GHX, Henry Schein Inc., Abbott, and CR Bard.

2) The medical device supply chain symposium held in Minneapolis provided a forum for medical device companies, government representatives, and academia to discuss critical supply chain management issues and look for opportunities for standard development and research. 
Manufacturing Metrology and Standards for the Health Care Enterprise Program report

\subsection{Bioimaging}

\section{Introduction and goals}

Medical imaging is a multibillion dollar industry and is making major strides into current medical diagnosis and therapy. The market in 2004 (source: Business Communications Co., Inc, www.bccresearch.com) for imaging equipment, picture archiving and communication systems, and computer-aided detection systems was $\$ 7.9$ billion dollars with an average annual growth rate (AAGR) of 5.5\%. According to this report "the fastest growing category of the imaging market consists not of imaging equipment, but of computer systems that are used as adjuncts to imaging equipment, which is expected to grow at a $14.9 \%$ AAGR." Key technical barriers that are hampering progress in medical imaging are: 1) lack of standards for data collection and analysis across an array of different commercial imaging platforms including associated metadata; 2) lack of validated and robust software methods for measurement of change in computed extracted features such as tumor volume or other anatomical, functional or metabolic metrics, 3) inadequate physical performance standards and related image quality standards particularly for qualifying image attributes for measuring change; and 4) lack of technical interoperability standards for ensuring standard image rendering, related meta data, data storage and retrieval.

To address the above barriers NIST has embarked on a bioimaging initiative as part of NIST's American Competitive Initiative (ACI) program, which will continue beyond the conclusion of the MEL healthcare program. One of the components of the bioimaging initiative is "Standards and Validation for Software," which is being undertaken by ITL (Information Technology Laboratory) and MEL. The goal of the MEL project is to improve the quality of image acquisition, analysis, and storage through effective standardization, improved software, and rigorous testing. We have been working closely with NIH (NCI, NIBIB and NCRR), Pharma, FDA, and other concerned government agencies and industry representatives such as NEMA to achieve our goals. The project is intended to support clinical decision making by highlighting deviations from normal conditions by image analysis of diseased organs and to suggest potential disease conditions. The intention is not to provide an automated diagnosis. While providing a general framework for building clinical decision support systems through image analysis, the initial project focuses on images captured through wireless endoscopy of the gastrointestinal system. The framework will then be extended to the lung cancer databases.

\section{Technical overview}

There are several stages to the image analysis project:

1) Collect images with metadata that includes information about modality, spatial resolution, compression, and other attributes in a Health Insurance Portability and Accountability Act (HIPAA) format

2) Develop a representation scheme for image ontologies

3) Develop an image analysis program for extracting features and

4) Test and validate. 
The sequence of steps for Stage 3, starting from image analysis to identification of a diagnostic suggestion, is:

1) Image segmentation. The partitioning of a digital image locates a set of regions that collectively cover the image. The pixels in a region are similar with respect to some visual properties, such as color, intensity or texture while adjacent regions are significantly different. The segmentation method and the feature selection described below are organ and modality specific.

2) Feature vector computation. Sets of numbers are calculated respectively for each image region. These numbers, called features, describe a variety of region properties in numerical form.

3) Mapping into disease ontology. Mapping is performed by comparing feature vectors with predefined vectors associated with specific nodes in the ontology representing disease conditions and vectors associated with normal conditions.

4) Highlighting lesions. The region with feature vectors similar to the feature vectors of known lesions is highlighted so as to attract the attention of the physician.

5) Diagnostic suggestion. If a given region is similar to a known lesion, a suggestion of a diagnosis can be provided to the physician.

Testing and validation will be done by our industry affiliates at Texas Tech University Health Sciences Center and Asian Institute of Gastroenterology.

\section{Achievements}

Achievements include:

1) A workshop was held at NIST in 2006 to determine the need to standardize imaging methods for data collection and data analysis in the context of drug or radiation therapy trials. The NIST meeting was a stakeholders meeting attended by $230+$ scientists from academia, imaging and pharmaceutical companies, contract research and trade organizations, representatives from imaging societies, and agencies of the Federal Government.

2) The image analysis experiment is being performed with video records from Wireless Capsule Endoscopy (WCE), a revolutionary technique which allows the visualization of the entire gastrointestinal (GI) tract. Our current project focuses on two aspects: discovering lesions and aiding in diagnosis. Our aim is not to replace a trained specialist but help him/her during the examination of the recorded video. The image segmentation and feature vector extraction components of the system are becoming mature and more robust. The disease mapping and diagnostic components are just beginning to take shape.

\section{Impacts}

The bioimaging workshop report has generated considerable interest in the radiology community. The Radiological Society of North America is planning on implementing some of the recommendations of the workshop. The image analysis work has elicited 
interest in applying the methodology to large databases of medical images such as the RIDER (Reference Image Database to Evaluate Response) project.

\section{Concluding comments}

The original goal of the program has been to apply proven MEL manufacturing technology and expertise to healthcare systems, biomedical devices and equipment, and biomedical data management. MEL provided a small amount of seed funding each year, with additional funding obtained from the Department of Health and Human Services and the American Competitiveness Initiative. We believe that the program was extremely successful in achieving its goals. Additionally, the knowledge gained in some of the projects, such as ADF, will allow us to develop similar frameworks in the manufacturing domain.

Disclaimer: No approval or endorsement of any commercial product by the National Institute of Standards and Technology is intended or implied. Certain commercial equipments, instruments, or materials are identified in this report to facilitate better understanding. Such identification does not imply recommendations or endorsement by the National Institute of Standards and Technology nor does it imply the materials or equipment identified are necessarily the best available for the purpose. 
Appendix. Project participants and outputs (project leads are identified by PL)

\begin{tabular}{|c|c|c|c|c|c|}
\hline \multicolumn{6}{|c|}{ 1. MEDICAL DEVICE PROJECTS } \\
\hline Project & $\begin{array}{l}\text { 1.1 Mobility } \\
\text { devices }\end{array}$ & 1.2 Hearing devices & $\begin{array}{l}1.3 \text { Surface } \\
\text { characterization of } \\
\text { biomedical devices }\end{array}$ & $\begin{array}{c}1.4 \text { Intelligent } \\
\text { assistive surgical } \\
\text { devices }\end{array}$ & $\begin{array}{c}\text { 1.5 Nano-bio } \\
\text { devices }\end{array}$ \\
\hline Participants & $\begin{array}{l}\text { - Roger } \\
\text { Bostelman (PL) } \\
\text { - James Albus } \\
\text { - U. of Pittsburgh } \\
\text { - U. of Delaware } \\
\text { - Florida Gulf } \\
\text { Coast } \\
\text { - Veterans } \\
\text { Administration } \\
\text { - Robotic } \\
\text { Rehabilitation } \\
\text { Resources }\end{array}$ & $\begin{array}{l}\text { - Victor Nedzelnitsky } \\
\text { (PL) } \\
\text { - Randall P. Wagner } \\
\text { (PL) } \\
\text { - Dept. of Veterans } \\
\text { Affairs (VA) } \\
\text { - Members of ANSI } \\
\text { Accredited (ASA) } \\
\text { Standards } \\
\text { Committee S3 } \\
\text { Bioacoustics and its } \\
\text { Working Groups, } \\
\text { especially S3/WG48 } \\
\text { Hearing Aids }\end{array}$ & $\begin{array}{l}\text { - Theodore } \\
\text { Vorburger (PL) } \\
\text { - Alan Zheng } \\
\text { - Lisa Pakstis } \\
\text { - Joy Dunkers } \\
\text { - Marcus Cicerone } \\
\text { - Timothy Quinn }\end{array}$ & $\begin{array}{l}\text { - Nicholas } \\
\text { Dagalakis (PL) } \\
\text { - Yongsik Kim } \\
\text { - Damien Bertot } \\
\text { - Junhwan Kim } \\
\text { - D. Sawyer } \\
\text { - S. Phillips } \\
\text { - Bruce Borchard } \\
\text { - Dana } \\
\text { Strawbridge } \\
\text { - Marvin } \\
\text { Kingsbury }\end{array}$ & $\begin{array}{l}\text { - John A. Dagata } \\
\text { (PL) } \\
\text { - Natalia Farkas } \\
\text { - Bob Shull } \\
\text { - Cindi Dennis } \\
\text { - Vince Hackley } \\
\text { - Esther Chang } \\
\text { - Kathleen } \\
\quad \text { Pirollo }\end{array}$ \\
\hline Outputs & 1) through 10) & 33) through 40) & 11) & 12), 13) & 14) through 24) \\
\hline
\end{tabular}




\begin{tabular}{|c|c|c|c|c|c|}
\hline \multicolumn{6}{|c|}{ 2. HEALTHCARE INFORMATICS PROJECTS } \\
\hline Project & $\begin{array}{l}2.1 \text { Clinical } \\
\text { informatics }\end{array}$ & 2.2 Bioinformatics & $\begin{array}{c}2.3 \text { Enterprise } \\
\text { modeling and } \\
\text { simulation }\end{array}$ & $\begin{array}{c}\text { 2.4 Manufacturing } \\
\text { and value chain } \\
\text { management }\end{array}$ & 2.5 Bioimaging \\
\hline Participants & $\begin{array}{l}\text { - Steven Fenves } \\
\text { (PL) } \\
\text { - E. Subrahmanian } \\
\text { (PL) } \\
\text { - Puja Goyal } \\
\text { - Jean-Cyrus } \\
\text { Angbo } \\
\text { - Fauzi Daoud } \\
\text { - Ram Sriram } \\
\text { - ONCHIT }\end{array}$ & $\begin{array}{l}\text { - Ram Sriram } \\
\text { (PL) } \\
\text { - E. Subrahmanian } \\
\text { - Antonio Cardone } \\
\text { - Soo-Yong Shin } \\
\text { - Penn. State U. }\end{array}$ & $\begin{array}{l}\text { - Charles McLean } \\
\text { (PL) } \\
\text { - Damien Bertot } \\
\text { - Sanjay Jain } \\
\text { - Tina Lee } \\
\text { - Swee Leong } \\
\text { - Guodong Shao } \\
\text { - Benjamin Raverdy }\end{array}$ & $\begin{array}{l}\text { - Shaw Feng (PL) } \\
\text { - U of. Minnesota }\end{array}$ & $\begin{array}{l}\text { - Ram Sriram (PL) } \\
\text { - Steven Fenves } \\
\text { - E. Subrahmanian } \\
\text { - Mala Ramaiah } \\
\text { - Marcin Kociolek } \\
\text { - Texas Tech UHS } \\
\text { - AIG }\end{array}$ \\
\hline Outputs & $25), 26)$ & $27), 28)$ & 29), 30), 31) & & 32) \\
\hline
\end{tabular}




\section{References}

1) Bostelman, R., Albus, J., "Survey of Patient Mobility and Lift Technologies Toward Advancements and Standards," NISTIR 7384, National Institute of Standards and Technology, Gaithersburg, MD, 2006.

2) Bostelman, R., Albus, J., "HLPR Chair - A Service Robot for the Healthcare Industry," 3rd International Workshop on Advances in Service Robotics, Vienna, Austria, July 7, 2006.

3) Bostelman, R., Russo, P., Albus, J., Hong., T., Madhavan, R., "Applications of a 3D Camera Towards Healthcare Mobility Aids," IEEE International Conference on Networking, Sensing and Control, Ft. Lauderdale, FL, April 2006.

4) Bostelman, R., Albus, J., Recent Developments of the HLPR Chair, International Conference on Rehabilitation Robotics (ICORR 2007), Noordwijk aan Zee, The Netherlands, June 13-15, 2007.

5) Bostelman, R., J. Albus, J.,HLPR Chair: A Novel Indoor Mobility-Assist and Lift System, ASME 2007 International Design Engineering Technical Conferences \& Computers and Information in Engineering Conference, Las Vegas, NV, September 4-7, 2007.

6) Bostelman, R., Albus, J., A Novel Patient Mobility and Rehabilitation Robot, Presented at the 13th IASTED International Conference on Robotics and Applications 2007 (RA07), Wurzburg, Germany, August 29-31, 2007.

7) Bostelman, R., Albus, J.,A Multipurpose Robotic Wheelchair and Rehabilitation Device for the Home, 2007 IEEE/RSJ International Conference on Intelligent Robots and Systems (IROS) San Diego, CA, Oct 29 - Nov 2, 2007.

8) Bostelman, R., Albus, J., Hong, T., Madhavan, R., Applications of a 3D Range Camera Towards Healthcare Mobility Aids, IEEE ICNSC Conf., Ft. Lauderdale, FL, April 2006.

9) Bostelman, R., Albus, J., Design of the HLPR Chair, NISTIR 7459, National Institute of Standards and Technology, Gaithersburg, MD, September 2007.

10) Bostelman, R., Albus, J., Robotic Patient Lift and Transfer, Book Chapter in I-Tech new book project "Service Robotics," to be published March 2008.

11) Pakstis, L. M., Dunkers, J. P., Zheng, A., Vorburger, T., Quinn, T. and Cicerone, M., "Optimization of Cellular Response on Flexible Surfaces Using Chemically Bound Proteins," submitted for publication in Biomaterials.

12) Dagalakis, N. G., Kim, Y.-S., Sawyer, D. and Shakarji, C. "Development of Tools for Measuring the Performance of Computer Assisted Orthopaedic Hip Surgery Systems," PerMIS '07, Gaithersburg, MD, August 28-30, 2007.

13) (http://www.isd.mel.nist.gov/medical_devices/PerMIS_OR_CAOHS_Artfct_Vs32.pd f) 
14) Farkas, N., Comer, J. R., Zhang, G., Evans, E. A., Ramsier, R. D., and Dagata, J. A., "SPM oxidation and parallel writing on zirconium nitride thin films,"J. Vac. Sci. Technol. A 23 846, 2005.

15) Farkas, N., Comer, J. R., Zhang, G., Evans, E. A., Ramsier, R. D., and Dagata, J. A., "High voltage SPM oxidation of ZrN: Materials for multiscale applications," Nanotechnology 16 262, 2005.

16) Chien, F.S., Hsieh, W.F., Gwo, Jun, J. S., Silver, R. M., Vladar, A. E. and Dagata, J.A., "Characterization of Prototype Silicon Pitch artifacts by Scanning Probe Oxidation and Anisotropic wet Etching," J Vac. Sci. Technol. B, 23 66, 2005.

17) Farkas N., Ehrman J.D., Evans E. A., Ramsier R. D., Dagata J. A., "High-voltage parallel writing on iron nitride thin films," JVSTA 24 1340, 2006.

18) Rodriguez, O., Fricke, S., Chien, C., Dettin, L., VanMeter, J., Shapiro, E., Dai, H.N., Casmiro, M., Ileva, I., Dagata, J., Johnson, J., Koretsky, A. and Albanese, C., "Contrast-enhanced in vivo imaging of cancer cells by MRI," Cell Cycle, 5 113, 2006.

19) Pirollo, K. P., Dagata, J., Wang, P., Freedman, M., Vladar, A., Fricke, S., Ileva, L., Zhou, Q., and Chang, E. S., "A tumor-targeted nanodelivery system to improve early MRI detection of cancer," Molecular Imaging 5 41, 2006.

20) Dagata, J. A., "Fundamental science and lithographic applications of scanning probe microscope oxidation," in S. Kalinin and A. Gruverman, Scanning probe microscopy: Electrical and electromechanical phenomena at the nanoscale, (Springer, NY 2006) p. 858.

21) Pirollo, K. F., Rait, A., Zhou, Q., Hwang, S., Dagata, J. A., Zon, G., Hogrefe, R. I., Palchik, G. and Chang, E. H., "Materializing the potential of small interfering RNA via a tumor-targeting nanodelivery system," Cancer Research 67 2938, 2007.

22) Kuramochi H., Tokizaki T., Dagata J.A. and Yokoyama H., "Why nano-oxidation with carbon nanotube probes is so stable. I. Linkage between hydrophobicity and stability," Nanotechnology 18 135703, 2007.

23) Kuramochi H., Tokizaki T., Ando K., Yokoyama H. and Dagata J.A., "Why nanooxidation with carbon nanotube probes is so stable. II. Bending behaviour of CNT probes during nano-oxidation," Nanotechnology 18 135704, 2007.

24) Yu, W., Pirollo, K. F., Dagata, J. A. Rait, A., Yu, B., Huang, W. and Chang, E. H., "Structural differences between oligopeptides influence the biological activity of nanoimmunoliposome complexes," submitted to Nucleic Acids Research.

25) Bock, C., Carnahan, L., Fenves, S. J., Gruninger, M., Kashyap, V., Lide, B., Nell, J., Raman, R. and Sriram, R. D., "Healthcare Strategic Focus Area: Clinical Informatics, "NISTIR 7263, National Institute of Standards and Technology, Gaithersburg, MD, 2005. 
26) Fenves, S. J., Subrahmanian, E., Goyal, P., Angbo, J.-C., Daoud, F. and Sriram, R. D., "The Architecture Development Facilitator (ADF): First year report," NISTIR 7411, National Institute of Standards and Technology, Gaithersburg, MD, 2007.

27) Ravichandran, V. and Sriram, R.D., "Toward Data Standards for Proteomics," Nature Biotechnology, Volume 23, Number 3, pages 373-376, March 2005.

28) Shin, S-Y., Kim, S., Eom, J-H., Zhang, B-T. and Sriram, R.D., “Identifying ProteinProtein Interaction Sentences Using Boosting and Kernel Methods," Second BioBreAtivE Challenge Workshop - Critical Assessment of Information Extraction in Molecular Biology, Madrid, Spain, 2007.

29) Jain, S. and Mclean, C., "Simulation Applications in Health Care: A Survey of Recent Advances," submitted for publication.

30) Shao, G. and Lee, T., "Applying Software Product Line Technology to Simulation Modeling of Emergency Response Facility," under review by Journal of Defense Modeling and Simulation.

31) Jain, S., "Movement in emergency room of a hospital using the High Level Architecture IEEE 1516," 2006 Winter Simulation Conference.

32) Clarke, L. and Sriram, R.D., "Imaging as a Biomarker: Standards for Change Measurements in Therapy", Proceedings of the United States Measurement System (USMS) Workshop, NIST, Gaithersburg, MD, September 14-15, 2006, 2007.

\section{Standards published by committees in $\mathbf{w}$ hich program members participated}

1) ANSI S3.35-2004 Method of Measurement of Performance Characteristics of Hearing Aids Under Simulated Real-Ear Working Conditions

2) IEC 60118-13 (2004-11): Electroacoustics - Hearing aids - Part 13: Electromagnetic compatibility (EMC).

3) IEC 60118-7 (2005-10): Electroacoustics - Hearing Aids - Part 7: Measurement of the performance characteristics of hearing aids for production, supply and delivery quality assurance purposes.

4) IEC 60118-8 (2005-10): Electroacoustics - Hearing Aids - Part 8: Methods of measurement of performance characteristics of hearing aids under simulated [in situ] working conditions.

5) IEC 60118-4 (2006-10): Electroacoustics - Hearing aids - Part 4: Induction loop systems for hearing aid purposes - Magnetic field strength.

6) IEC 60645-5 (2004-11): Electroacoustics - Audiometric equipment - Part 5: Instruments for the measurement of aural acoustic impedance/admittance.

7) IEC 60645-3 (2007-03): Electroacoustics - Audiometric equipment - Part 3: Test signals of short duration. 
8) IEC 60318-5 (2006-08): Electroacoustics - Simulators of human head and ear - Part 5: $2 \mathrm{~cm}^{3}$ coupler for the measurement of hearing aids and earphones coupled to the ear by means of ear inserts.

\section{Acronyms}

AAGR

$\mathrm{ACI}$

$\mathrm{AD}$

$\mathrm{ADF}$

AIG

ANSI

ASA

ASTM

$\mathrm{BD}$

CAOS

CIP

DLS

DNA

E-A-R ${ }^{\circledR}$

ebXML

EBM

EDI

EHCR

EHR

FDA

FIRST/FRODA

FY

GDP

GHX

GI

GUMC

HHS
Average Annual Growth Rate

American Competitive Initiative

Alzheimer's Disease

Architecture Development Facilitator

Asian Institute of Gastroenterology

American National Standards Institute

Acoustical Society of America

American Standards for Testing of Materials

Becton, Dickinson and Company

Computer Assisted Orthopedic Surgery

Cdk5 Inhibitor Protein

Dynamic Light Scattering

Deoxyribose Nucleic Acid

A brand name for Aero Corp.'s foam insert ear plugs

Electronic Business using eXtensible Markup Language

Evidence-Based Medicine

Electronic Data Interchange

Efficient Healthcare Consumer Response

Electronic Health Record

Food and Drug Administration

Floppy Inclusions and Rigid Substructure Topography/

Framework Rigidity Optimized Dynamics Algorithm

Fiscal Year

Gross Domestic Product

Global Healthcare Exchange

Gastrointestinal

Georgetown University Medical Center

Department of Health and Human Services 
Manufacturing Metrology and Standards for the Health Care Enterprise Program report

HIBCC

HIPPA

HJ-CAOHS

HLPR

HMC

ICDR

ID

IEC

IOM

ISD

ISO/IEC

IT

ITL

MDDI

MEL

MFM

MRI

MSEL

$\mathrm{NCI}$

NCL

NCRR

NDS

NEMA

NHIN

NIBIB

NIST

$\mathrm{NIH}$

NLS

OEM
Health Industry Business Communications Council

Health Insurance Portability and Accountability Act

Horizontal Joint-Computer Assisted Orthopedic Hip

Surgery

Home Lift, Position, and Rehab

Hershey Medical Center

Interagency Committee on Disability Research

Identification

International Electrotechnical Commission

Institute of Medicine

Intelligent Systems Division

International Organization for Standardization /

International Electrotechnical Commission

Information Technology

Information Technology Laboratory

Medical Device and Diagnostic Industry

Manufacturing Engineering Laboratory

Magnetic Force Microscopy

Magnetic Resonance Imaging

Material Science and Engineering Laboratory

National Cancer Institute

Nanotechnology Characterization Laboratory

National Center for Research Resources

Nanoparticle Drug Delivery Systems

National Electrical Manufacturers Association

Nationwide Health Information Network

National Institute of Biomedical Imaging and Bioengineering

National Institute of Standards and Technology

National Institutes of Health

National Library Service

Original Equipment Manufacturer 
$\mathrm{ONC}$

ONCHIT

PIE

PL

PPI

RIDER

SB

SB\&M

SE

SM

SPIO

SPM

SQUID

SVM

TATRC

UBL

UHS

UN/EDIFACT

USMS

USNC/IEC

VA

VCAT

W3C

WCE

XML
Office of the National Coordinator for Health Information Technology

Office of the National Coordinator for Health Information Technology

Protein Interaction information Extraction system

Project Lead

Protein-Protein Interaction

Reference Image Database to Evaluate Response

Systems Biology

Systems Biology and Medicine

Systems Engineering

Systems Medicine

Super-Paramagnetic Iron-Oxide

Scanning Probe Microscopy

Superconducting Quantum Interference Devices

Support Vector Machine

Telemedicine and Advanced Technology Research Center

Universal Business Language

University Health Services

United Nations Directories for Electronic Data Interchange for Administration, Commerce, and Transport

United States Measurement System

United States National Committee of the International Electrotechnical Commission

Veterans Affairs

Visiting Committee on Advanced Technology

World Wide Web Consortium

Wireless Capsule Endoscopy

Extensible Markup Language 